\title{
Intrinsically Polar Piezoelectric Self-Assembled Oligopeptide Monolayers
}

Christopher A. Petroff Giuseppe Cassone Jiři Šponer Geoffrey R. Hutchison*

C. A. Petroff, Prof. G. R. Hutchison

Department of Chemistry, University of Pittsburgh, 219 Parkman Avenue, Pittsburgh, Pennsylvania 15260, United States

Email Address: geoffh@pitt.edu

Dr. G. Cassone

Institute for Chemical-Physical Processes, National Research Council, Viale F. Stagno d'Alcontres 37, 98158 Messina, Italy

Prof. J. Šponer

Institute of Biophysics of the Czech Academy of Sciences, Královopolská 135, 61265 Brno, Czech Republic

Prof. G. R. Hutchison

Department of Chemical and Petroleum Engineering, University of Pittsburgh, 3700 O'Hara Street, Pittsburgh, Pennsylvania 15261, United States

Keywords: Piezoelectricity, Peptide, Poling free, SAM, Piezoelectric Voltage Constant

Flexible, bio-compatible piezoelectric materials are of considerable research interest for a variety of applications, but many suffer from low response or high cost to manufacture. Herein, novel piezoelectric force and touch sensors based on self-assembled monolayers of oligopeptides are presented which produce large piezoelectric voltage response and are easily manufactured without the need for electrical poling. While the devices generate modest piezoelectric charge constants $\left(d_{33}\right)$ of up to $9.8 \mathrm{pC} \mathrm{N}^{-1}$, they exhibit immense piezoelectric voltage constants $\left(g_{33}\right)$ up to $2 \mathrm{~V} \mathrm{~m} \mathrm{~N}^{-1}$. Furthermore, a flexible device prototype is demonstrated that produces open-circuit voltages of nearly $6 \mathrm{~V}$ under gentle bending motion. Improvements in peptide selection and device construction promise to further improve the already outstanding voltage response and open the door to numerous practical applications.

Piezoelectric materials find use in a wide range of applications from touch and vibration sensors ${ }^{[1]}$ to energy harvesters ${ }^{[2]}$ to micromechanical actuators. ${ }^{[3]}$ These devices rely on the piezoelectric effect to interconvert mechanical stress and electrical charge. In the direct piezoelectric effect, an applied force produces a resultant charge, whereas, in the converse effect, an applied voltage causes a mechanical deformation. Most existing piezoelectric materials are hard, brittle, leadcontaining ceramics such as lead zirconium titanate (PZT). ${ }^{[4]}$ As such, these materials have limited ranges of motion, are liable to crack, and are not bio-compatible. While there is a large research focus on developing flexible, bio-compatible piezoelectric materials, ${ }^{[5]}$ much of this work has involved placing traditional piezoelectrics on or into flexible substrates, often sacrificing electrical performance for added flexibility and ease of manufacturing (i.e., $d$-values $<200 \mathrm{pC} \mathrm{N}^{-1}$ instead of $500 \mathrm{pC} \mathrm{N}^{-1}-600 \mathrm{pC} \mathrm{N}^{-1}$ for PZT). ${ }^{[2,6,7]}$ In addition to well known piezoelectric polymers such as semi-crystalline poly(vinylidene fluoride) (PVDF), researchers have begun to develop fundamentally new piezoelectric materials such as helicenes, amino acids, viruses, and peptides. ${ }^{[5,8-12]}$ 
While the most commonly reported piezoelectric constant is the piezoelectric charge constant, $d$, the piezoelectric voltage constant, $g$, is perhaps more meaningful for sensing applications, since large voltages can be easily and accurately detected. ${ }^{[1,13-15,11]}$ Despite its importance, there are few examples of the voltage constant reported in the literature, making device comparisons difficult (see Guerin et al. ${ }^{[15]}$ and Chen et al. ${ }^{[16]}$ as examples). When both polarization and stress are along the Z-axis, the subscript 33 is used (i.e., $d_{33}$ and $g_{33}$ ). The piezoelectric charge and voltage constants are related by the relative permittivity such that $g_{33}=\frac{d_{33}}{\varepsilon_{r} \varepsilon_{0}}$, where $\varepsilon_{r}$ is the relative permittivity of the material and $\varepsilon_{0}$ is the vacuum permittivity. ${ }^{[17]}$ As such, low dielectric constant organics have the potential to produce high-response piezoelectric sensors. ${ }^{[15]}$ The piezoelectric voltage constant is often calculated from the piezoelectric charge constant, or it can be measured directly from applied stress and resulting electric field. Direct measurements - while lacking in the literature - are perhaps more meaningful for low-frequency sensing applications such as from human touch, as conversions are often calculated using the relative permittivity for a higher frequency, which may or may not translate to real-world use at lower frequencies. The relative permittivity of many piezoelectric polymer composites is shown to increase at lower frequencies, ${ }^{[18-20]}$ which would lead to a decreased piezoelectric voltage constant at those frequencies.

Piezoelectricity occurs naturally in the body and plays an important role in processes such as bone growth. ${ }^{[5]}$ This phenomenon has allowed for the production of piezoelectric nanogenerators made from tissue harvested from both plants ${ }^{[21,22]}$ and animals. ${ }^{[23,24]}$ While it has been demonstrated that amino acids and peptides are piezo-active, ${ }^{[5]}$ significant challenges exist in applying them to usable devices, including aligning the materials or crystals to produce a bulk piezoelectric response by means of an external high-voltage electric field. ${ }^{[5]}$

We find self-assembled monolayers (SAMs) to be a promising approach to bulk alignment of piezoelectric molecules ${ }^{[25,26]}$ and thus present piezoelectric SAM (PSAM) devices based on selfassembled oligopeptide monolayers. SAMs have been widely studied, and thiol-containing molecules are known to form uniform, stable monolayers on gold substrates. ${ }^{[27]}$ Self-assembled piezoelectric devices present significant advantages over more traditional approaches as they are intrinsically polar and can be easily produced through solution processing.

SAMs of thiol-containing oligopeptides, ranging in length from seven to thirteen amino acids 
A

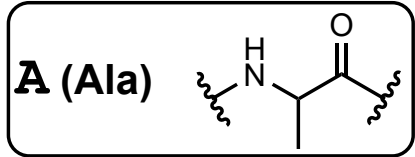

$\mathbf{C A}_{6} \overbrace{\mathrm{NH}_{2}}^{\mathrm{O}}$<smiles>[R]C(=O)C(CS)N[Y12]#[Z12][H]</smiles>

$\mathrm{CA}_{9}$<smiles>[R]COC(=O)C(N)CS</smiles>

$\left(\mathrm{CAx}-\mathrm{NH}_{2}\right)$ $\xi \mathrm{NH}_{2}$

$R=$

Carboxylate

Terminated

$\left(C A_{X}\right)$<smiles></smiles>

Amide

Terminated
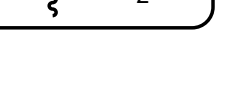

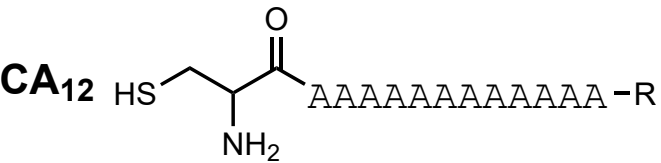

Figure 1: Generalized scheme outlining PSAM devices studied. (A) Chemical structures of peptides used. (B) 3D structure of $\alpha$-helical peptide $\mathrm{CA}_{12}$ (top). Schematic diagram showing an applied force compressing a PSAM device, changing the dipole moment, and leading to the buildup of charge and subsequent flow of current (bottom).

were formed from solution on the gold-coated surface of printed circuit boards (PCBs). The peptide sequences, shown in Figure 1(A), consist of cysteine $(\mathrm{C})$ and six, nine, or twelve alanines $(\mathrm{A})$; both carboxylate-terminated and amide-terminated forms were studied for most sequences. These sequences were chosen as they are short, helical, thiol-containing oligopeptides that are similar except for their varying lengths and end groups. As illustrated in Figure 1(B), assemblies of a peptide functionalized PCB facing a polyurethane (PU) coated PCB (with optional 1-dodecanethiol (DDT) coating) were tested in a quasi-static manner using an automated system. Piezoelectric constants were subsequently calculated from these data. Moreover, state-of-the-art ab initio molecular dynamics (AIMD) simulations were carried out in order to help interpret, on a microscopic basis, some of the experimental results.

The peptides used are helical and should act as "molecular springs" [28,29,25] when compressed, leading to much greater length changes than similar linear molecules such as the DDT used as a control. As the length of each peptide changes, so too does its dipole; therefore, when a force is applied to compress these "springs," charge builds up on the surface, leading to a measurable piezoelectric response. ${ }^{[28,29,25]}$ The piezoelectric charge constant $\left(d_{33}\right)$ is calculated by integrating the measured current and plotting the resultant charge versus the applied force (Figure S1, Supporting 
Information); since there is no convention for defining the positive $Z$-axis in our self-assembled, non-crystalline materials, we used the absolute value of charge. Figure 2(A) and (B) presents a summary of $d_{33}$ values obtained for our PSAM devices. Computed $d_{33}$ values for peptide assemblies are consistently higher than those of the alkanethiol controls (Figure S3, Supporting Information). A small piezoelectric response is expected for DDT because SAMs contain an interface and are, therefore, inherently piezoelectric. ${ }^{[30]}$ A maximum value of $(9.8 \pm 1.5) \mathrm{pC} \mathrm{N}^{-1}$ is obtained for the assembly consisting of amide-terminated $\mathrm{CA}_{6}$ functionalized PCBs opposing PU coated DDT functionalized PCBs (CA6-NH2/DDT-PU). On average, peak current values of $80 \mathrm{pA}-100 \mathrm{pA}$ were observed at the maximum applied force of $\sim 6 \mathrm{~N}$. On average, the PSAM device assemblies had a resistance of $(56.9 \pm 1.8) \mathrm{M} \Omega$ and a capacitance (measured at $\sim 0.7 \mathrm{MHz})$ of $(428 \pm 14) \mathrm{pF}$; the only observed trend is that, generally, the capacitance slightly increased with increased chain length.

We experimentally examined several variations of our PSAM devices and used analysis of variance (ANOVA) to look and see how and if these changes affect the piezoelectric response in a statistically significant fashion (see Tables S1-S12, Supporting Information). Firstly, we varied the PCB opposing the peptide PCB in the device. These PCBs were coated in PU, and we looked at both unfunctionalized and DDT functionalized versions. With nearly double the response, peptides tested against PCBs where the PU layer coats a DDT monolayer (average $d_{33}$ of $7.9 \mathrm{pC} \mathrm{N}^{-1}$ ) produced statistically higher responses than peptides tested against PCBs where the PU layer coats bare gold (average $d_{33}$ of $4.1 \mathrm{pC} \mathrm{N}^{-1}$ ). While this difference in piezoelectric response is somewhat unexpected, we theorize the DDT may affect the organization and properties of the PU layer, thereby altering the piezoelectric responses of the devices.

Next, we analyzed the difference in piezoelectric response between carboxylate-terminated and amide-terminated peptides. The amide-terminated peptides appear to produce $(\mathrm{P}$-value $<0.05)$ statistically greater piezoelectric responses than carboxylate-terminated peptides do; this is counter intuitive at first, as the amide-terminated form should have a smaller dipole moment, but the amide-termination may also affect the tilt angle of the SAM or the stability and rigidity of the $\alpha$-helix. If the amide causes the SAM to stand more perpendicular to the surface, this can counteract the effect of a smaller dipole by increasing the effective dipole in the $Z$-direction. Additionally, if the $\alpha$-helices of the amide-terminated peptides are less rigid, they will deform more easily under 

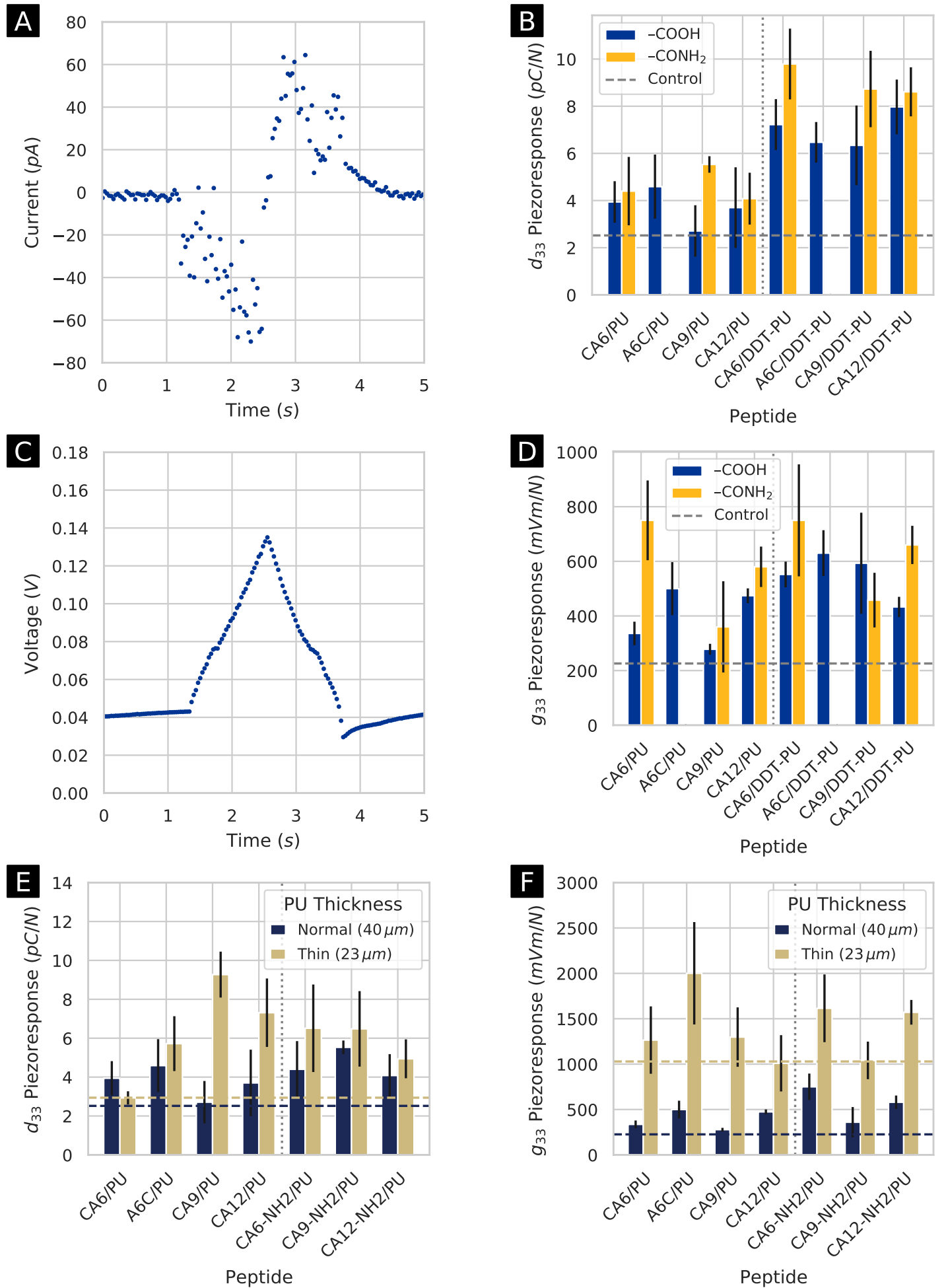

Figure 2: Piezoelectric response. (A) Representative short-circuit current measurement for one compression of a PSAM device. (B) The piezoelectric charge constants $\left(d_{33}\right)$ of PSAM devices containing PU coated DDT PCBs are greater than those containing PU coated unfunctionalized PCBs. Amide-terminated peptides present higher responses than carboxylate-terminated ones. Peptide responses are greater than those of DDT controls (dashed line; see Figure S3, Supporting Information). (C) Representative open-circuit voltage measurement for one compression of a PSAM device. Note that the baseline has been corrected. (D) The piezoelectric voltage constants $\left(g_{33}\right)$ of PSAM devices. Peptide responses are greater than those of DDT controls (dashed line; see Figure S4, Supporting Information). Error bars represent standard error across multiple samples. (E) The piezoelectric charge constants $\left(d_{33}\right)$ of PSAM devices using normal thickness PU $(40 \mu \mathrm{m})$ compared with those using thinner PU $(23 \mu \mathrm{m})$. (F) The piezoelectric voltage constants $\left(g_{33}\right)$ of PSAM devices using normal thickness PU compared with those using thinner PU. The dashed lines show the piezoelectric response of DDT controls (see Figure S3 and S4, Supporting Information) where navy corresponds to the normal thickness and gold corresponds to the thinner thickness. 
compression, leading to a greater change in dipole and, therefore, greater piezoelectric response.

Finally, we looked at varying the peptide sequence to alter its length; we tested $\mathrm{CA}_{6}, \mathrm{~A}_{6} \mathrm{C}, \mathrm{CA}_{9}$, and $\mathrm{CA}_{12}$ sequences. Somewhat surprisingly, the length of the peptide did not statistically alter the measured response despite the length-dictated dipole differences. Several explanations exist for the analogous values: they are similar because the longer peptides may not stand as straight on the surface, leading to a lower response in the $Z$-direction; the SAMs of the longer peptides may pack less densely, leading to lower response per unit area; and/or the responses are dominated by the hydrogen bonding of the $\alpha$-helices of the peptide backbone, which may be invariant of the peptide length. Indeed, previous computational work in our group on similar peptides showed no clear length effect on piezoelectric response. ${ }^{[31]}$

We turned to AIMD simulations to help explain our experimental results. We examined the carboxylate-terminated $\mathrm{CA}_{6}$ sequence and the corresponding amide-terminated $\mathrm{CA}_{6}-\mathrm{NH}_{2}$ sequence. The modeling shows that the piezoelectric responses of our peptides are largely dependent on the strength of the hydrogen bonds in the $\alpha$-helices of the peptide backbones. The backbone of the carboxylate-terminated peptides was found to be more stable and rigid than its amide-terminated counterpart. Furthermore, higher applied electric fields were needed before the carboxylateterminated peptide deformed. These data agree favorably with our experimental results, which show that the amide-terminated peptides produce higher response than the carboxylate-terminated peptides and suggest that the length of the peptide plays a relatively minor role in the overall piezoelectric response. For a more detailed, quantitative discussion of the AIMD results, see the Supporting Note and Figure S8-S11 (Supporting Information).

Our PSAM devices show great potential as piezoelectric sensors as demonstrated by their high piezoelectric voltage constants $\left(g_{33}\right)$. These voltage constants are calculated by plotting induced electric field (measured voltage divided by sample thickness) against external stress (applied force divided by sample area) and determining the linear fit (Figure S2, Supporting Information). As shown in Figure 2(C) and (D), $g_{33}$ values up to $(750 \pm 150) \mathrm{mV} \mathrm{m} \mathrm{N}^{-1}$ were obtained; for comparison, this is an order of magnitude greater than the $g_{33}$ value of $<40 \mathrm{mV} \mathrm{m} \mathrm{N}^{-1}$ for $\mathrm{PZT}^{[32,33]}$ and is also greater than the predicted $g_{33}$ value of $\sim 480 \mathrm{mV} \mathrm{m} \mathrm{N}^{-1}$ for a racemic alanine crystal ${ }^{[11]}$. As expected, the measured $g_{33}$ values are greater than those obtained for alkanethiol controls (Figure 
S4, Supporting Information). While the oft reported peak voltage produced by piezoelectric devices is important for showing their potential practical use, the voltage value is affected by many factors including the device area and thickness as well as the force applied, making it difficult to compare devices reported in the literature. For example, we saw, on average, a peak voltage of $\sim 0.2 \mathrm{~V}$ at the maximum applied force of $6 \mathrm{~N}$, but if we had only applied $3 \mathrm{~N}$ of force, the maximum voltage would have been merely $\sim 0.1 \mathrm{~V}$. In contrast, the piezoelectric voltage constant $(g)$ - the voltage analog of the ubiquitous piezoelectric charge constant $(d)$-allows for meaningful comparisons of sensing potential but is, unfortunately, largely absent from the literature.

While we initially planned to examine the voltage response of our PSAM devices analogously to our approach for the charge response, the results are more varied and less conclusive. Although the same trends - greater DDT-PU response, greater amide-termination response, and no length effect-are present, they are not statistically significant. To help explain this incongruity, we examined the difference in response between individual PU and DDT-PU PCBs. While the different PCBs within each category were statistically similar for values of the piezoelectric charge constant, the piezoelectric voltage constant values obtained for individual DDT-PU PCBs were statistically different. This lack of uniformity is likely because capacitive and leakage effects hold a greater role in the voltage measurements and quite possibly vary PCB to PCB due to defects in the dielectric layer.

The induced electric field plays a critical role in the magnitude of the piezoelectric voltage constant; it is dependent on both the measured voltage and the device thickness. For our PSAM devices, we calculated the induced electric field based on the distance between the electrodes; this distance is almost entirely dictated by the thickness of our PU dielectric layer, whereas the absolute voltage should be largely independent of thickness. Accordingly, we looked to increase our induced electric field and piezoelectric voltage constant by decreasing the PU thickness. We accomplished this by diluting our uncured PU with petroleum ether before spin-coating and drying. When we tested our peptides against these thinner dielectric layers, we observed a much greater piezoelectric voltage response (Figure 2(E) and (F)); unexpectedly, we also saw an increase in the piezoelectric charge response. It is unknown how the petroleum ether used to reduce the PU thickness affected the spin coating, drying, and final properties of the PU dielectric layer; the PCBs 
with the thinner PU coating were less consistent, as we observed statistical differences between the individual PCBs in both the piezoelectric charge and voltage constants. We suspect that changes to the PU dielectric layer resulted in the statistically greater piezoelectric charge constant $\left(d_{33}\right)$ values for PSAM devices containing these thinner PU PCBs; the charge constant should be largely independent of the thickness of the dielectric layer. Nonetheless, the $g_{33}$ values obtained for thin PU PSAM devices are considerably greater than the change in $d_{33}$ values alone can account for. Since the thin PU layer is nearly half the thickness of the normal PU layer, we expect the resultant PSAM devices (including DDT controls) to have almost twice the voltage response. Indeed, the $g_{33}$ values of up to $(2000 \pm 600) \mathrm{mV} \mathrm{m} \mathrm{N}^{-1}$ observed for the thin PU PSAM devices agree, within error, to those expected from the combination of the $d_{33}$ increase and the PU thickness decrease; on average, the $g_{33}$ values for the PSAM devices containing PCBs with the thinner PU coating were $(220 \pm 40) \%$ greater than those with the normal thickness PU, whereas the expected increase based on the combination of $d_{33}$ increase $(60 \pm 30) \%$ and thickness decrease $(74 \pm 2) \%$ is $(180 \pm 60) \%$. We suspect that the PU dielectric layer is key to the devices' large voltage responses as it allows for the large voltages to be produced without shorting across the SAM. The maximum $g_{33}$ value of $2 \mathrm{~V} \mathrm{~m} \mathrm{~N}^{-1}$ is quite remarkable and, to the best of our knowledge, is the highest experimental value reported to date in the literature.

The long-term stability of piezoelectric devices is of importance to their practical adoption; as such, we measured the stability of the piezoelectric response of our devices in multiple ways. Our PSAM devices show remarkable stability and retain their initial piezoelectric response for at least three months when stored away from light in a vacuum desiccator (Figure S5, Supporting Information); this is in line with the expected stability of SAMs under these storage conditions. While the long-term storage stability is adequate, the response of our normal PSAM devices decays over a matter of hours when exposed to ambient conditions. In order to solve this problem, we produced sealed PSAM devices where we placed the two PCBs together before the PU dried. The active layer of these sealed PSAM devices is protected from the atmosphere. When we tested these devices, we found that, while the charge response was much lower, the voltage response was similar to that of our normal PSAM devices due to the thinner PU layer (Figure S6, Supporting Information). On the stability front, the response actually increased over several weeks of testing 
A

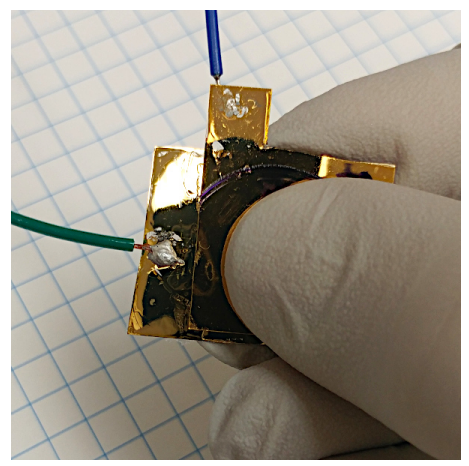

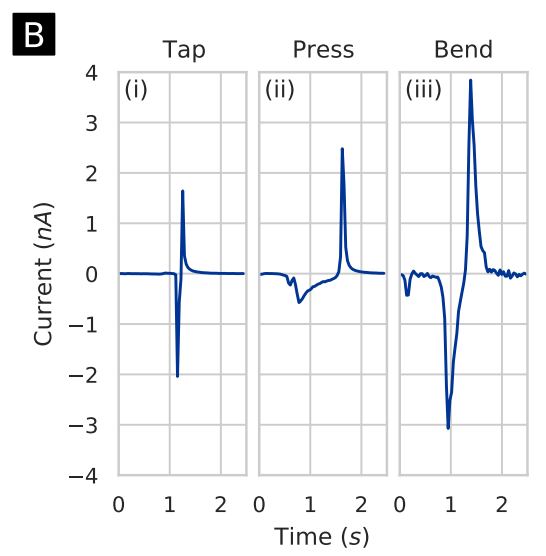

Figure 3: Flexible sealed PSAM device (CA12-NH2/DDT-PU) fabricated on gold-coated plastic substrates. (A) Image of device being tested. (B) Current response of flexible device. (C) Voltage response of flexible device. Current and voltage responses were measured due to (i) light finger taps while mounted, (ii) firm finger presses while mounted, and (iii) bending while held between three fingers. The device produced nearly $4 \mathrm{nA}$ of short-circuit current and $6 \mathrm{~V}$ of open-circuit voltage when subject to gentle bending motion.

(Figure S7, Supporting Information). Some of the observed increase in response is due to the slight decrease in preload force over time due to the nature of our testing setup; the rest of the increase might be due to organizing effects in the monolayer over time. These results show that, with improved manufacturing methods, our PSAM devices have real potential in practical applications.

To further demonstrate the potential practical application of our PSAM devices, we constructed a sealed device made using flexible, gold-coated substrates. When tested using our normal method, we measured a $d_{33}$ value of $(3.68 \pm 0.08) \mathrm{pC} \mathrm{N}^{-1}$ and a $g_{33}$ value of $(35 \pm 4) \mathrm{mVm} \mathrm{N}^{-1}$ (the PU layer is an order of magnitude thicker, but its thickness and uniformity are somewhat uncertain). In addition, we examined our flexible device under more practical conditions by measuring its current and voltage in response to finger taps, presses, and bends (Figure 3). The finger taps and presses led to maximum currents of approximately $2 \mathrm{nA}$ and voltages of approximately $4 \mathrm{~V}$, whereas the bends produced almost $4 \mathrm{nA}$ and $6 \mathrm{~V}$. These values are much greater than those obtained when the device is tested normally $(0.006 \mathrm{nA} / 0.06 \mathrm{~V})$ and are likely due to the more localized nature of the force. The measured voltage response is much greater than that of most flexible devices reported in the literature while simultaneously being considerably easier to manufacture, making it a formidable candidate for potential practical applications.

We present a innovative new method of producing thin, flexible, non-crystalline organic piezoelectric devices based on SAMs that show great potential for practical applications. The devices 
are simple, easy to manufacture due to their self-assembled nature that negates the need for electrical poling, and produce large voltage responses important for potential sensing applications. Furthermore, their peptide nature makes them fully biocompatible and easily modifiable. Future work in tuning the sequence and functionalization of the amino acids as well as use of better dielectrics and more precise manufacturing methods holds the potential for large increases to the already outstanding voltage response of our PSAM devices.

\section{Experimental Section}

Materials: Peptides $\mathrm{CA}_{6}$ and $\mathrm{A}_{6} \mathrm{C}$ were obtained from Sigma-Genosys. All other peptides were obtained from AnaSpec. Ethanol (200 proof) was obtained from Decon Labs. Acetonitrile ( $\geq 99.9 \%)$ and 1-dodecanethiol ( $\geq 98 \%$ ) were obtained from Sigma-Aldrich. Petroleum ether (certified ACS) was obtained from Fisher Scientific. Liquid conformal polyurethane coating was obtained from MG Chemicals (Urethane Conformal Coating; Cat. No. 4223-55ML). Spray conformal polyurethane coating was obtained from Techspray (Fine-L-Kote UR Conformal Coating; Cat. No. 2104-12S). All chemicals were used as received. Ultrapure water $(18.2 \mathrm{M} \Omega \mathrm{cm})$ was generated using a Millipore Synergy system.

Device preparation: Self-assembled monolayers (SAMs) were formed on the outer gold surface of $5 \mathrm{~cm} \times 5 \mathrm{~cm}$ custom designed and manufactured printed circuit boards (PCBs) (Where Labs/DirtyPCBs.com) with $3.5 \mathrm{~cm} \times 3.5 \mathrm{~cm}$ electroless nickel immersion gold (ENIG) finished copper pads. The PCBs were first cleaned by ultrasonicating them in ethanol for at least 30 min; rinsing sequentially with ethanol, water, and ethanol; and then drying them under a stream of nitrogen gas. Monolayers were formed by submerging the PCBs in a $0.5 \mathrm{~mm}-1 \mathrm{~mm}$ solution of the desired chemical or peptide for $48 \mathrm{~h}$ under ambient conditions to ensure uniformity. Solutions were prepared with either ethanol, water, acetonitrile, or a combination of the solvents depending on solubility; the solvent should not influence the resulting SAM and is not present in the final device. ${ }^{[27]}$ After SAM formation, PCBs were removed from solution and washed using the same procedure as above before being wrapped in aluminum foil and stored in a vacuum desiccator. Topography images taken with an atomic force microscope (AFM) (Asylum Research MFP-3D, 
AC mode, AC240TS-R3 cantilever) show SAM uniformity (Figure S12, Supporting Information). Wires were soldered onto the PCBs for testing.

In order to obtain consistent, reproducible contact between the PCBs, a commercial conformal polyurethane $(\mathrm{PU})$ coating was applied to the surface of some PCBs using a spin coater (Chemat Technology Spin Coater KW-4A, $1 \mathrm{~mL}$ PU, $1000 \mathrm{rpm}$ for $6 \mathrm{~s}$ increasing to $6000 \mathrm{rpm}$ for $10 \mathrm{~s})$. A thinner PU coating was obtained by mixing the PU with petroleum ether $(50 / 50 \mathrm{v} / \mathrm{v})$ before spin coating. The thickness of the PU coating was measured using calipers $(0.040 \pm 0.004$ mm normal coating; $0.023 \pm 0.003 \mathrm{~mm}$ thinner coating).

Sealed piezoelectric self-assembled monolayer (PSAM) devices were prepared by spraying one PCB with an aerosol can of commercial conformal PU, placing the other PCB and a $1 \mathrm{~kg}$ weight on top, and allowing the PU to dry. The PU thickness is $\sim 0.01 \mathrm{~mm}$. A flexible sealed PSAM device was prepared by depositing a $10 \mathrm{~nm}$ titanium adhesion layer followed by a $100 \mathrm{~nm}$ gold layer on flexible Nunc Thermanox Plastic Coverslips (Thermo Scientific) using an electron beam evaporation system (Plassys Electron Beam Evaporator MEB550S); the flexible sealed PSAM device was then prepared similarly to the PCB sealed PSAM devices except that liquid PU was drop cast to form the dielectric layer $(\sim 0.42 \mathrm{~mm}$ thickness). The flexible sealed PSAM device has an active area of $2.5 \mathrm{~cm} \times 2.5 \mathrm{~cm}$.

Device testing and characterization: Samples were removed from the desiccator at least $1 \mathrm{~h}$ prior to testing, as inconsistent results were obtained when testing was performed sooner. PSAM devices consisting of one PU coated PCB facing one uncoated, SAM functionalized PCB were tested in a quasi-static manner before the piezoelectric response was calculated. Similar to our previous work, ${ }^{[34]}$ the PSAM device was positioned in a testing apparatus where force was applied using a stepper motor controlled threaded rod. A force sensor (Tekscan FlexiForce A201), with a poly(dimethylsiloxane) spacer on top, rested between the rod and the device under test. In order to reduce triboelectric charge generation, a preload force of $\sim 1 \mathrm{~N}$ was applied using the threaded rod before compressions of varying force (up to $\sim 6 \mathrm{~N}$ ) were applied along the Z-axis at a rate of approximately $0.17 \mathrm{~mm} \mathrm{~s}^{-1}$. Force and short-circuit current or open-circuit voltage measurements were recorded for $70 \mathrm{~s}$ and $90 \mathrm{~s}$, respectively, using a Keithley 2614B SourceMeter. Each recorded measurement is the average of the values computed from three sequential, undisturbed 
test sequences, and each sample was tested at least five times with the PCBs of the PSAM device separated between measurements; sealed PSAM devices were not separated, but the preload force was removed and reapplied. As reported in our previous work, the system was tested on commercial ceramic piezoelectric materials to ensure accuracy. ${ }^{[34]}$

The collected data are simply the applied force and measured current or voltage- both as a function of time. To calculate the piezoelectric charge constant $\left(d_{33}\right)$, force versus charge was plotted for each compression and the slope of the linear best fit was calculated using a robust linear regression (Figure S1, Supporting Information). A custom Python script was used to compute charge by integrating the current peaks over time; ${ }^{[34]}$ we modified our previous script to optimize the peak finding sensitivity and to use a robust linear regression. The script identifies force peaks and subtracts off any baseline force; it then looks for the corresponding current peak and integrates it to calculate the resultant charge during the period of increasing applied force. The piezoelectric voltage constant $\left(g_{33}\right)$ was calculated in a similar manner except that, after the force and voltage peaks were identified, voltage was converted to induced electric field by dividing by sample thickness and force was converted to external stress by dividing by electrode area (see Figure S2 and minimum working example Python script in Supporting Information). Resistance was measured using a Keithley 2614B SourceMeter. Capacitance was measured using a Zhengzhou Minghe Electronic Technology LC100-A inductance capacitance meter.

Ab initio molecular dynamics simulations: We used the software package CP2K, ${ }^{[35,36]}$ based on the Born-Oppenheimer approach, to perform ab initio molecular dynamics (AIMD) simulations of samples containing carboxylate-terminated peptide $\mathrm{CA}_{6}$ or amide-terminated peptide $\mathrm{CA}_{6}-\mathrm{NH}_{2}$ solvated in liquid water; both were under the action of static and homogeneous electric fields applied along a given direction (corresponding to the Z-axis). The implementation of an external field in numerical codes based on Density Functional Theory (DFT) can be achieved by exploiting the Modern Theory of Polarization and Berry's phases ${ }^{[37-39]}$ (see, e.g., Ref. ${ }^{[40]}$ ). The $\mathrm{CA}_{6}$-containing numerical sample was composed of one $\mathrm{CA}_{6}$ peptide solvated by $253 \mathrm{H}_{2} \mathrm{O}$ molecules (i.e., 833 atoms) arranged in a cubic cell with edge equal to $20.4 \AA$, so as to reproduce the liquid water experimental density of $1.00 \mathrm{~g} \mathrm{~cm}^{-3}$ at room temperature. Similarly, the $\mathrm{CA}_{6}-\mathrm{NH}_{2}$-containing numerical sample was composed of one $\mathrm{CA}_{6}-\mathrm{NH}_{2}$ peptide solvated by $253 \mathrm{H}_{2} \mathrm{O}$ molecules (i.e., 
835 atoms) arranged in a cubic cell with edge equal to $20.4 \AA$. As usual, in order to minimize nonphysical surface effects, all structures were replicated in space by employing periodic boundary conditions. The intensity of the electric field was gradually increased with a step increment of $0.5 \mathrm{~V}$ $\mathrm{nm}^{-1}$ from zero up to a maximum of $1.0 \mathrm{~V} \mathrm{~nm}^{-1}$. In the zero-field cases, we performed dynamics of 50 ps for each investigated sample whereas, for each other value of the field intensity, we ran dynamics of 20 ps, thus accumulating a global simulation time equal to 180 ps where a time-step of 0.5 fs was chosen. Additional tests employing different atomistic configurations of the initial structures and/or assigning diverse initial atomic velocities were executed in order to exclude biases stemming from specific initial molecular arrangements.

Wavefunctions of the atomic species were expanded in the triple-zeta valence plus polarization (TZVP) basis set with Goedecker-Teter-Hutter pseudopotentials using the GPW method. ${ }^{[41]} \mathrm{A}$ plane-wave cutoff of 400 Ry was imposed. Exchange and correlation (XC) effects were treated with the Becke-Lee-Yang-Parr (BLYP) ${ }^{[42]}$ density functional. Moreover, in order to take into account dispersion interactions, we employed the dispersion-corrected version of BLYP (i.e., BLYP-D3). [43,44] The adoption of the BLYP-D3 functional has been dictated by the widespread evidence that such a functional, when dispersion corrections are taken into account, offers one of the best adherences with the experimental results related to water among the standard GGA functionals. ${ }^{[45,46]}$ It is well-known that neglecting dispersion corrections leads to a severely over-structured liquid (see, e.g., Ref. [47] and references therein). In order to counteract the overstructuring of intermolecular interactions typically induced by GGA XC functionals, all simulations were executed at a temperature of $350 \mathrm{~K}$. The dynamics of nuclei were simulated classically within a constant number, volume, and temperature (NVT) ensemble using the Verlet algorithm whereas the canonical sampling was executed by employing a canonical-sampling-through-velocity-rescaling thermostat ${ }^{[48]}$ set with a time constant equal to 10 fs.

\section{Supporting Information}

Supporting Information is available from the Wiley Online Library or from the author.

\section{Acknowledgments}

The authors thank Z. N. Georgieva for assistance with spin coating and Dr. N. C. Miller for assistance in preparing the flexible substrates. The authors acknowledge the University of Pittsburgh for 
financial support. Software used in this work includes, in part, Avogadro, ${ }^{[49]} \mathrm{CP} 2 \mathrm{~K},{ }^{[50]}$ Matplotlib, ${ }^{[51]}$ NumPy, ${ }^{[52]}$ pandas, ${ }^{[53]}$ pySerial, SciPy, ${ }^{[54]}$ Statsmodels, ${ }^{[55]}$ and Python-VXI11.

\section{Author Contributions}

C.A.P. designed the testing system and carried out the experiments and data analysis. G.C. and

J.Š. designed the ab initio molecular dynamics simulations; G.C. executed the $a b$ initio molecular dynamics simulations. G.R.H conceived and directed the project. All authors wrote and reviewed the manuscript.

\section{Conflict of Interest}

The authors declare no conflict of interest.

\section{References}

[1] J. F. Tressler, S. Alkoy, R. E. Newnham, J. Electroceram. 1998, 2, 4257.

[2] J. Briscoe, S. Dunn, Nano Energy 2015, 1415.

[3] K. Uchino, J. Electroceram. 2007, 20, 3-4 301.

[4] K. S. Ramadan, D. Sameoto, S. Evoy, Smart Mater. Struct. 2014, 23, 3033001.

[5] D. Kim, S. A. Han, J. H. Kim, J.-H. Lee, S.-W. Kim, S.-W. Lee, Adv. Mater. (Weinheim, Ger.) 2020, 32, 141906989.

[6] C. Dagdeviren, P. Joe, O. L. Tuzman, K.-I. Park, K. J. Lee, Y. Shi, Y. Huang, J. A. Rogers, Extreme Mech. Lett. 2016, 9269 .

[7] H. Li, C. Tian, Z. D. Deng, Appl. Phys. Rev. 2014, 1, 4041301.

[8] O. Stetsovych, P. Mutombo, M. Švec, M. Šámal, J. Nejedlý, I. Císařová, H. Vázquez, M. MoroLagares, J. Berger, J. Vacek, I. G. Stará, I. Starý, P. Jelínek, J. Am. Chem. Soc. 2018, 140, 3 940.

[9] J.-H. Lee, K. Heo, K. Schulz-Schönhagen, J. H. Lee, M. S. Desai, H.-E. Jin, S.-W. Lee, ACS Nano 2018, 12, 88138.

[10] E. S. Hosseini, L. Manjakkal, D. Shakthivel, R. Dahiya, ACS Appl. Mater. Interfaces 2020, 12, 89008 .

[11] S. Guerin, J. O’Donnell, E. U. Haq, C. McKeown, C. Silien, F. M. F. Rhen, T. Soulimane, S. A. M. Tofail, D. Thompson, Phys. Rev. Lett. 2019, 122 047701.

[12] B. Y. Lee, J. Zhang, C. Zueger, W.-J. Chung, S. Y. Yoo, E. Wang, J. Meyer, R. Ramesh, S.-W. Lee, Nat. Nanotechnol. 2012, 7, 6351.

[13] E. K. Akdogan, M. Allahverdi, A. Safari, IEEE Trans. Ultrason. Ferroelectr. Freq. Control 2005, 52, 5746.

[14] A. Safari, E. K. Akdogan, Ferroelectrics 2006, 331, 1153. 
[15] S. Guerin, A. Stapleton, D. Chovan, R. Mouras, M. Gleeson, C. McKeown, M. R. Noor, C. Silien, F. M. F. Rhen, A. L. Kholkin, N. Liu, T. Soulimane, S. A. M. Tofail, D. Thompson, Nature Mat. 2017, 17, 2180.

[16] X. Chen, H. O. T. Ware, E. Baker, W. Chu, J. Hu, C. Sun, Procedia CIRP 2017, 65157 , 3rd CIRP Conference on BioManufacturing.

[17] ANSI/IEEE Std 176-1987 1988, 1-66.

[18] B. P. Kumar, H. Kumar, D. Kharat, Mater. Sci. Eng., B 2006, 127, 2130 .

[19] W. Wan, J. Luo, C. e Huang, J. Yang, Y. Feng, W.-X. Yuan, Y. Ouyang, D. Chen, T. Qiu, Ceram. Int. 2018, 44, 55086.

[20] A. Zak, W. Gan, W. A. Majid, M. Darroudi, T. Velayutham, Ceram. Int. 2011, 37, 51653.

[21] S. Bairagi, S. Ghosh, S. W. Ali, Sci. Rep. 2020, 10, 1.

[22] S. Maiti, S. Kumar Karan, J. Lee, A. Kumar Mishra, B. Bhusan Khatua, J. Kon Kim, Nano Energy 2017, 42 282 .

[23] S. K. Ghosh, D. Mandal, Appl. Phys. Lett. 2016, 109, 10103701.

[24] S. K. Ghosh, D. Mandal, Appl. Phys. Lett. 2017, 110, 12123701.

[25] X. Quan, J. D. Madura, G. R. Hutchison, Self-assembled monolayer piezoelectrics: Electric-field driven conformational changes, 2017, URL https://arxiv.org/abs/1706.08993.

[26] N. C. Miller, H. M. Grimm, W. S. Horne, G. R. Hutchison, Nanoscale Adv. 2019, 14834.

[27] J. C. Love, L. A. Estroff, J. K. Kriebel, R. G. Nuzzo, G. M. Whitesides, Chem. Rev. 2005, 105, 41103.

[28] X. Quan, C. W. Marvin, L. Seebald, G. R. Hutchison, J. Phys. Chem. C 2013, 117, 3316783.

[29] C. W. Marvin, H. M. Grimm, N. C. Miller, W. S. Horne, G. R. Hutchison, J. Phys. Chem. B 2017, 121, 4410269.

[30] M.-M. Yang, Z.-D. Luo, Z. Mi, J. Zhao, S. P. E, M. Alexe, Nature 2020, 584, 7821377.

[31] N. N. D. Gayatri, G. Hutchison, Origins of negative and positive electromechanical response of oligopeptide piezoelectrics, 2019, URL https://doi.org/10.26434/chemrxiv.9985205.v1.

[32] H. D. Chen, K. R. Udayakumar, L. E. Cross, J. J. Bernstein, L. C. Niles, J. Appl. Phys. (Melville, NY, U. S.) 1995, 77, 73349.

[33] W. Goh, K. Yao, C. Ong, Appl. Phys. A: Mater. Sci. Process. 2005, 81, 51089.

[34] C. A. Petroff, T. F. Bina, G. R. Hutchison, ACS Appl. Energy Mater. 2019, 2, 96484.

[35] J. Hutter, M. Iannuzzi, F. Schiffmann, J. VandeVondele, Wiley Interdiscip. Rev.: Comput. Mol. Sci. 2014, 4, 115.

[36] J. VandeVondele, M. Krack, F. Mohamed, M. Parrinello, T. Chassaing, J. Hutter, Comput. Phys. Commun. 2005, 167, 2103.

[37] R. D. King-Smith, D. Vanderbilt, Phys. Rev. B 1993, 471651.

[38] R. Resta, Rev. Mod. Phys. 1994, 66899. 
[39] M. V. Berry, Proc. R. Soc. London, Ser. A 1984, 392, 180245.

[40] P. Umari, A. Pasquarello, Phys. Rev. Lett. 2002, 89157602.

[41] M. Krack, Theor. Chem. Acc. 2005, 114, 1-3 145.

[42] A. D. Becke, Phys. Rev. A 1988, 383098.

[43] S. Grimme, J. Antony, S. Ehrlich, H. Krieg, J. Chem. Phys. 2010, 132, 15154104.

[44] S. Grimme, S. Ehrlich, L. Goerigk, J. Comput. Chem. 2011, 32, 71456.

[45] I.-C. Lin, A. P. Seitsonen, I. Tavernelli, U. Rothlisberger, J. Chem. Theory Comput. 2012, 8, 103902.

[46] A. Bankura, A. Karmakar, V. Carnevale, A. Chandra, M. L. Klein, J. Phys. Chem. C 2014, 118, 5029401.

[47] M. J. Gillan, D. Alfè, A. Michaelides, J. Chem. Phys. 2016, 144, 13130901.

[48] G. Bussi, D. Donadio, M. Parrinello, J. Chem. Phys. 2007, 126, 1014101.

[49] M. D. Hanwell, D. E. Curtis, D. C. Lonie, T. Vandermeersch, E. Zurek, G. R. Hutchison, J. Cheminf. 2012, 4, 1.

[50] T. D. Kühne, M. Iannuzzi, M. Del Ben, V. V. Rybkin, P. Seewald, F. Stein, T. Laino, R. Z. Khaliullin, O. Schütt, F. Schiffmann, D. Golze, J. Wilhelm, S. Chulkov, M. H. Bani-Hashemian, V. Weber, U. Borštnik, M. Taillefumier, A. S. Jakobovits, A. Lazzaro, H. Pabst, T. Müller, R. Schade, M. Guidon, S. Andermatt, N. Holmberg, G. K. Schenter, A. Hehn, A. Bussy, F. Belleflamme, G. Tabacchi, A. Glöß, M. Lass, I. Bethune, C. J. Mundy, C. Plessl, M. Watkins, J. VandeVondele, M. Krack, J. Hutter, J. Chem. Phys. 2020, 152, 19194103.

[51] J. D. Hunter, Comput. Sci. Eng. 2007, 9, 390.

[52] S. Van Der Walt, S. C. Colbert, G. Varoquaux, Comput. Sci. Eng. 2011, 13, 222.

[53] W. McKinney, In Stéfan van der Walt, Jarrod Millman, editors, Proceedings of the 9th Python in Science Conference. 201056 - 61.

[54] P. Virtanen, R. Gommers, T. E. Oliphant, M. Haberland, T. Reddy, D. Cournapeau, E. Burovski, P. Peterson, W. Weckesser, J. Bright, S. J. van der Walt, M. Brett, J. Wilson, K. Jarrod Millman, N. Mayorov, A. R. J. Nelson, E. Jones, R. Kern, E. Larson, C. Carey, İ. Polat, Y. Feng, E. W. Moore, J. Vand erPlas, D. Laxalde, J. Perktold, R. Cimrman, I. Henriksen, E. A. Quintero, C. R. Harris, A. M. Archibald, A. H. Ribeiro, F. Pedregosa, P. van Mulbregt, SciPy 1.0 Contributors, Nat. Methods 2020, 17261.

[55] S. Seabold, J. Perktold, In Stéfan van der Walt, Jarrod Millman, editors, Proceedings of the 9th Python in Science Conference. $201092-96$. 


\section{Table of Contents}
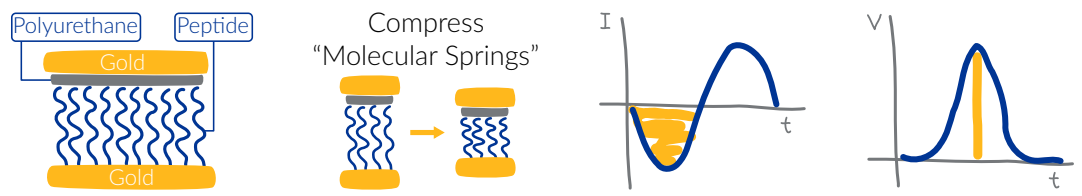

Novel, intrinsically polar oligopeptide based piezoelectric force and touch sensors producing large piezoelectric voltage response are presented. They are easily manufactured through solution processing, without the need for electrical poling. The devices exhibit immense piezoelectric voltage constants $\left(g_{33}\right)$ up to $2 \mathrm{~V} \mathrm{~m} \mathrm{~N}^{-1}$ and a flexible device prototype is demonstrated that produces open-circuit voltages of nearly $6 \mathrm{~V}$ under gentle bending motion. 